\title{
Rechtsgeschichte
}

http://www.rg-rechtsgeschichte.de/rg8

$\operatorname{Rg} 2006 \quad 70-83$

Zitiervorschlag: Rechtsgeschichte Rg 8 (2006)

http://dx.doi.org/10.12946/rg08/070-083

\section{Margrit Seckelmann}

\section{Im Labor}

Beobachtungen zum Rechtstransfer anhand des Europäischen Verfassungsvertrags 


\section{Abstract}

The European Constitutional Treaty and the European Charter of Fundamental Rights (Title II of the Constitutional Treaty) currently challenge the legal orders of the member states. Transfer processes are to be expected, e.g. due to the adoption of the "right to a good administration « by the European Charter of Fundamental Rights, the development of which will be presented in more detail. This paper tracks the way in which this legal principle developed from a structural condition for the granting of development aid ("good governance «) into a central category for the description of European minimum standards for government and administration. To provide some perspective, possible "reactions « by the legal orders of the member states, particularly of Germany and France, are sketched. Following up this point, considerations on the transfer of laws are pursued and a situational view of the communication among legal orders is advocated. In order to illustrate this approach, analogies between legal interactions and chemical reactions are drawn. 


\section{Im Labor}

Beobachtungen zum Rechtstransfer anhand des Europäischen Verfassungsvertrags

Können Rechtsprinzipien gleichsam explodieren? Betrachtet man die Diskussionen um die Konsequenzen aus dem französischen und niederländischen "Nein « zum europäischen Verfassungsvertrag, so kommen Analogien zu einem gescheiterten Experiment in den Sinn. Es scheint, als ob die normativen Vorstellungen des Verfassungskonvents sehr heftig mit der Rechtswirklichkeit reagiert hätten. Worin bestanden die Ausgangsstoffe für dieses Experiment? Der Vertrag über eine Verfassung für Europa enthält viele Destillate, welche aus den verschiedenen mitgliedstaatlichen Rechtsordnungen gewonnen wurden. Insbesondere gingen in die vorab verkündete und in den Titel II des Verfassungsvertrags übernommene Grundrechtecharta der Europäischen Union ${ }^{\mathrm{I}}$ Rechtsverbürgungen aus nationalstaatlichen Rechtsordnungen ein, welche als Teil der "gemeinsamen Verfassungsüberlieferungen der Mitgliedstaaten « gemäß Art. 6 Abs. 2 EU-Vertrag auch als allgemeine Grundsätze des Gemeinschaftsrechts gelten können. ${ }^{2}$

Derzeit stellt sich die Frage, ob aus den rauchenden Trümmern der europäischen Mehrebenenpolitik eine gemeinsame europäische Verfassung entstehen kann. Auch ist das Schicksal der EU-Grundrechtecharta noch ungeklärt. Wird diese torsohaft wie der Grundrechtekatalog der Paulskirche bestehen bleiben? Und welcher Rechtscharakter kommt ihr zu? Grundsätzlich hat die EU-Grundrechtecharta ohne die Inkorporation in den Verfassungsvertrag lediglich deklaratorischen Charakter. ${ }^{3}$ Dennoch ist zu vermuten, dass sie die Rechtsprechung des Europäischen Gerichtshofs nicht unbeeinflusst lassen wird. Die Rechtsprechung des EuGH wird die Grundrechtsverbürgungen der Charta mittelbar in das Primärrecht der EU überführen, ${ }^{4}$ wenn nicht ohnehin von einer faktischen Selbstbindung der an der Proklamation beteiligten Gemeinschaftsorgane einschließlich des Europäischen Rates auszugehen ist. ${ }^{5}$ Es kann zudem keinesfalls als ausgeschlossen gelten, dass sich das Bundesverfassungsgericht an diesen Grundsätzen orientieren wird. ${ }^{6}$ Wird es daher erneut der Rechtsprechung der Gerichtshöfe der EU obliegen, gleichsam den »Reaktionsbeschleuniger ${ }^{7}$ des Europäisierungsprozesses darzustellen, der anderes - wie die Rechtsprechung der nationalstaatlichen Gerichte - mitreißt ${ }^{8}$

In diesem Aufsatz soll der Frage nachgegangen werden, wie es zu der heftigen Reaktion zwischen der geplanten EU-Verfassung und der Verfassungswirklichkeit kam. Ist diese auf ein Transferproblem zurückzuführen? Kam es zu unvorhergesehenen Nebeneffekten bei der Destillation von Rechtsprinzipien oder bei ihrer Verbindung mit der Rechtswirklichkeit, ${ }^{9}$ die das Reagenzglas erhitzten, verrußten oder im schlimmsten Fall sogar sprengten?

Chemische Experimente entstehen aus Differenz. Stoffe reagieren um so heftiger, je unterschiedlicher ihre Ladung ist. Identität und Differenz des Stofflichen ermöglichen Explosionen, die zur Bildung neuer Rechtskonstruktionen führen. Aber wie lassen sich Identitäten und Differenzen feststellen? Und welcher Zeitpunkt wird gewählt, um diese Feststellung zu treffen? Spannung kann auch dann entstehen, wenn Rechtsprinzipien in eine veränderte Rechtswirklichkeit re-importiert werden. Ein solches Experiment soll im Folgenden näher betrachtet werden.
Vertrag über eine Verfassung für Europa, Teil II, Art. 99-I I4, Amtsblatt der Europäischen Union, 47. Jahrgang, I6. Dezember 2004, C 3 IO, $\mathrm{C}_{3}$ Io/5 I - $\mathrm{C}_{3 \mathrm{IO} /}$ 54; download unter http://europa.eu.int/eur-lex/lex/JOHtml. do?uri=OJ:C:2004:3 I0:SOM: DE:HTML (einges. am 8.I2.2005).

\footnotetext{
2 Magiera (2003) I 29; Wolf (2002); SCHILLING (2000); TETTINGER (200I).

3 Iber (2002) 493; DutZler, LukascheK, VRanes, Weidel (200I).

4 Mahlmann (2000) $425 \mathrm{f}$.

5 Magiera (2003) I 29.

6 MAgIERA (2003) I 29.

7 Zum Konzept von Recht als

»Reaktionsbeschleuniger "

SeCKELMANN (2006b) 349-352.
}

8 GORNIG, TRÜE (2000).

9 Aus der umfangreichen Literatur $\mathrm{zu}$ den "unintended side effects " von Regulierung sei beispielhaft erwähnt: COASE (I960); BöHRET, KonZendorf (I997). 
Im Labor: Versuchsanordnung

Der zu analysierende Versuch betrifft die europäische Mehrebenenpolitik ebenso wie die Folgen des Kolonialismus - mit anderen Worten: die rechtlichen Konsequenzen der Globalisierung. Untersucht werden soll, welche Dynamik die Destillation von Rechtsprinzipien entwickeln kann, und zwar nicht nur für die Stoffe, mit denen das Destillat in Verbindung kommt, sondern auch für diejenigen, aus denen es gewonnen wird. Die Beobachtung beginnt zeitlich mit dem Ende des Kolonialismus und endet mit dem Europäischen Verfassungsvertrag.

Ein explanans für die Ablehnung des Verfassungsvertrags in den Mitgliedstaaten der Europäischen Union ist das Problem der Legitimität. Dieses bezieht sich nicht nur auf die allgemeine Frage nach der Begründung und der Einhegung der Kompetenzen der Europäischen Union. ${ }^{\text {IO }}$ Es betrifft auch die Strategien, die zur Bewältigung dieser Problematik gewählt wurden, nämlich die Kodifikation des Grundrechteteils des Verfassungsvertrags, der als Grundrechtecharta der Europäischen Union bereits zuvor verabschiedet worden war. Sowohl der Verfassungsvertrag als auch die Grundrechtecharta der Europäischen Union waren in den Staaten, in denen die ablehnenden Referenden durchgeführt wurden, intensiv gelesen und besprochen worden. Dennoch scheint insbesondere in Frankreich eine profunde Skepsis zurückgeblieben zu sein, ob die in der Grundrechtecharta und im Grundrechteteil des Verfassungsvertrags enthaltenen Rechtsverbürgungen tatsächlich mit dem status quo der nationalen Grund- und Freiheitsrechte zu vergleichen seien.

Diese Befürchtung war nicht ganz unberechtigt, wie an einem Beispiel näher demonstriert werden soll. Analysiert werden soll das in Art. II
IOI des Verfassungsvertrags der Europäischen Union gewährte Recht "auf eine gute Verwaltung (Good Administration). Nach Art. 4I der Grundrechtecharta der Europäischen Union beziehungsweise der wortgleichen Formulierung in Art. II IOI des Verfassungsvertrags der Europäischen Union hat jeder Bürger eines EU-Mitgliedstaats gegen diesen Staat einen Anspruch auf Sicherung eines Mindestbestands von Rechten. $\mathrm{Zu}$ diesem gehören die Wahrung der körperlichen Unversehrtheit, des Eigentums und auch gewisser Prozessgrundrechte wie der fairness des Verfahrens. Letztere werden in Art. II IOI des EU-Verfassungsvertrags wie auch in Art. 4I der EU-Grundrechtecharta unter den weiteren Begriff der Good Administration subsumiert. Der ganzheitliche Begriff einer qualitativ als "gut « vorgegebenen Verwaltung erfasst über die Garantie von Prozessgrundrechten hinaus auch die Legitimität des politischen Systems, die Transparenz der Findung staatlicher Entscheidungen, die Integrität, Effizienz, Kohärenz und Anpassungsfähigkeit des öffentlichen Sektors an neue Herausforderungen sowie die Partizipation und Konsultation der Bürger und schließlich die Existenz eines Staatshaftungsanspruchs.

Der Begriff der Good Administration emergierte ebenso wie der mit ihm verwandte, ganzheitliche Begriff der Good Governance aus Auflagen, welche die so genannten »entwickelten « Staaten für die institutionelle Gestaltung der Empfängerstaaten von Entwicklungshilfe, ihren früheren Kolonien, machten. Dem Begriff war eine rasante Karriere beschieden, so dass die Staaten der früheren Kolonialherrn schließlich ihre eigene Verfasstheit einem gesteigerten demokratischen Legitimationsbedürfnis ausgesetzt sahen. Letztlich ist also zu analysieren, wie Prinzipien vor allem des angloamerikanischen Rechts

Io Zur komplexen Frage der »abgeleiteten Legitimität « der Europäischen Union vgl. statt vieler: SCHROEDER (I998); BENZ (200I) $283 \mathrm{f}$. 
über den Weg der Vergabebedingungen der Weltbank für Kredite und Gelder zur Entwicklungszusammenarbeit schließlich auch normierende Funktion für das deutsche, französische und sonstige nationalstaatliche Recht $\mathrm{zu}$ entfalten drohten. Das Gefühl der Verunsicherung gerade der deutschen und französischen Bürger lässt sich auch damit erklären, dass diese angesichts der begrifflichen Vagheit des »Rechts auf eine gute Verwaltung « - und seiner »potentielle[n] Endoffenheit " ${ }^{\mathbf{I}}$ - einen Rückbau rechtsstaatlicher Grundsätze, etwa einer Abkehr von generell-abstrakten Regeln zu konkreten Fällen und den Rückbau der klaren Trennung von Justiz und Verwaltung befürchteten. So wurde namentlich das deutsche Rechtsstaatsprinzip von Robert von Mohl, ${ }^{\text {I2 }}$ Otto Bähr, ${ }^{\text {I3 }}$ Rudolf von Gneist ${ }^{\text {I4 }}$ und anderen aus einer Gesamtschau und immer weiter gehenden Abstraktion von englischen Prozessgrundrechten und französischen Verfassungsprinzipien entwickelt. ${ }^{{ }^{\mathrm{I}}}$

Wie ist nun der »Re-Import « von Grundsätzen zu beurteilen, aus denen das Rechtsstaatsprinzip seinerzeit entwickelt wurde? Kann dieser die Verwaltungsverfahrensrechte der Mitgliedstaaten irritieren, kommt es darüber hinaus vielleicht sogar zu einer Erschütterung der verfassungsrechtlichen Garantien, etwa der Grundrechte? Die Skepsis gegenüber dem Verfassungsvertrag für die Europäische Union scheint darauf hinzudeuten, dass dies in den Mitgliedstaaten zumindest teilweise befürchtet wird. Allerdings lassen sich im Bereich der europäischen Mehrebenenpolitik viele Transferprozesse dogmatischer Figuren aus einer mitgliedstaatlichen Verwaltungsrechtsordnung in eine andere beobachten. Das gilt auch für die »Exporte « von dogmatischen Figuren des deutschen öffentlichen Rechts, etwa die Übernahme des deutschen Verhältnismäßigkeitsgebots in die Gemeinschafts- rechtsordnung und von dort aus in das englische Public Law. ${ }^{\mathrm{I6}}$

Ist die Sicherung gewisser Grundrechtsstandards, wie sie das deutsche Bundesverfassungsgericht in seinen "Solange -Entscheidungen $^{17}$ und dem Maastricht-Urteil ${ }^{\text {I8 }}$ formuliert hat, mit anderen Worten ein Versuch kultureller Hegemonie der deutschen Akteure in der Europäischen Union - und für die französischen ließe sich Ähnliches nachweisen -, der den Export rechtsdogmatischer Figuren nur in einer Richtung zulässt?

\section{Die Debatte}

Die Übernahme von Rechtsinstituten aus anderen Rechtsordnungen ist - auch angesichts der Rechtsgestaltung durch die Organe der Europäischen Gemeinschaften - derzeit stark in der Diskussion. Es stehen sich, wie Martin de Jong, Virginie Mamadouh und Konstantinos Lalenis jüngst festgestellt haben, im Prinzip zwei Positionen gegenüber: Die evolutionäre Perspektive geht davon aus, dass sich Rechtsinstitute historisch entwickeln. Diese Position betrachtet die Verpflanzung von Instituten aus anderen Rechtssystemen mit Misstrauen. ${ }^{\text {I9 }}$ Manche, wie Pierre Legrand, bringen es dahingehend auf den Punkt, dass eine "Transplantation « von Rechtsinstituten schlechthin nicht möglich sei: Das Wort »Brot« könne, so führt er unter Bezug auf das berühmte Diktum Walter Benjamins aus, im Deutschen schließlich auch nicht mit dem französischen Begriff "pain « bezeichnet werden, ohne größte Verwirrung auszulösen. ${ }^{20}$ Eine entgegengesetzte Perspektive sieht derartige »Transplantationen « deutlich optimistischer: Eine Institution sei ein soziales Konstrukt, welches durch Gesetzgeber, Verwaltung, Rechtsdogmatik

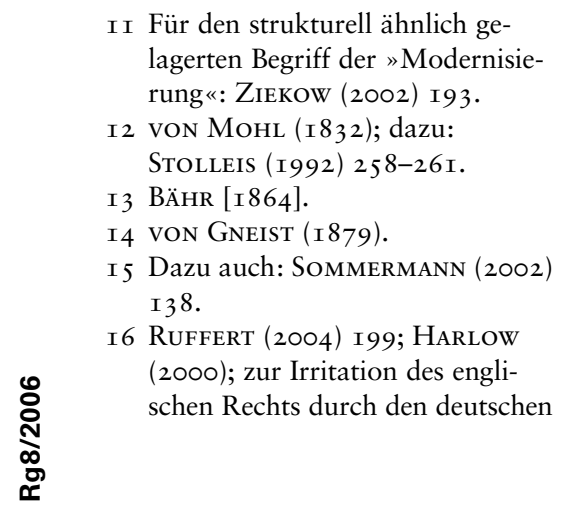

I Für den strukturell ähnlich gelagerten Begriff der "Modernisierung «: ZIEKOW (2002) I93.

MoHL (I832); dazu:

3 BÄHR [I 864].

4 VON GNEIST (I 879).

5 Dazu auch: SOMMERMANN (2002)

I38.

(2000); zur Irritation des engli-

schen Rechts durch den deutschen

Grundsatz von Treu und Glauben

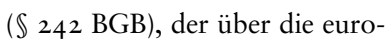
päische Richtlinie zum Verbraucherschutz Eingang in das common law fand, vgl. Teubner (200I) $35 \mathrm{I}$.

I7 BVerfG - II. Senat vom 29. Mai I 974 - BvL 52/7I, BVerfGE 37, 27I (Solange I); BVerfG - II. Senat vom 22. Oktober 1986 -, 2 BvR 197/83, BVerfGE 73, 339 (Solange II).
I 8 BVerfG - II. Senat vom I 2. Oktober 1993-2BvR 2I34, 21 59/92, BVerfGE 89, I 55 (Maastricht).

I9 LEgRAND (I997) i i7; BENJAMin (I977) 55 .

20 De Jong, Mamadouh, Lalenis (2002) 283. 
oder Rechtsprechung auch radikal geändert werden könne. ${ }^{2 I}$ In seinem viel diskutierten Buch über Legal Transplants nimmt Alan Watson eine solche Haltung ein: Rechtstransplantationen habe es immer gegeben, und diese seien auch die fruchtbarste Quelle für eine Rechtsentwicklung. ${ }^{22}$ Die transferpessimistische Perspektive, insbesondere auch das genannte Beispiel, sei zu simplifizierend, denn die jeweilige Entwicklung von Recht und von Brot weise durchaus ihre Eigengesetzlichkeiten auf. ${ }^{23}$

Watsons Art der Beschreibung von Transferprozessen orientiert sich an den Leitdifferenzen »innen/außen « einer Rechtsordnung sowie "notwendig/kontingent " einer Übernahme. ${ }^{24}$ Marie Theres Fögen und Gunther Teubner haben die »klassische « Erklärung der Rezeption des Römischen Rechts in Deutschland anhand dieser Leitdifferenzen untersucht. Sie sind zu dem Schluss gekommen, dass seit dem titelgebenden Werk Georg von Belows die Ursachen der Rezeption des römischen Rechts in Deutschland in einer fehlenden Kraft des deutschen Rechts zur Evolution gesucht worden seien. Nach dieser Erzählung habe das deutsche Recht zu seiner Weiterentwicklung exogener Einflüsse bedurft. ${ }^{25}$ Den teleologischen »Meistererzählungen ${ }^{26}$ entgegen haben sie daher vorgeschlagen, eine zielorientierte Betrachtungsweise bei der Darstellung von Transferprozessen zu vermeiden und vielmehr von spezifischen Formen einer »Episodenverknüpfung « zu sprechen, also von einer situativen Verbindung rekursiver Operationen. Interaktionen des Rechtssystems mit außerrechtlichen Bereichen seien anhand der Leitdifferenz »enge Kopplung/lose Kopplung von Recht und Sozialbereich « besser zu erklären als mit den herkömmlichen Kategorien. ${ }^{27}$

Eine situative Betrachtungsweise von wechselseitigen Beeinflussungsprozessen soll auch hier gewählt werden. Rechtsprinzipien und »Lebensverhältnisse « können unter bestimmten Bedingungen aufeinander »reagieren «. Die Übernahme eines Rechtsinstituts aus einer anderen Rechtsordnung kann dabei - und hier kann man durchaus an von Below anknüpfen - als Katalysator, als Reaktionsbeschleuniger wirken. ${ }^{28}$ Ich möchte daher ein Bild von Josef Kohler benutzen, um die Übernahme von dogmatischen Figuren aus fremden Rechtsordnungen zu beschreiben:

"Wie es nun Sache des chemischen Forschers ist, durch richtige Vereinigung von chemischen Stoffen neue Verbindungen herzustellen, und wie gerade die erfinderische Kraft darin beruht, daß derselbe aus der Unzahl der Möglichkeiten solche Combinationen wählt, aus welchen neue fruchtbare Gebilde hervorzugehen scheinen: so ist es Sache des dogmatischen Juristen, ein Princip in der Fülle der Lebensverhältnisse einzutauchen und ihm gerade diejenigen Verhältnisse zu nähern, in welchen das Prinzip seine interessantesten Bildungen zu Stande bringt. ${ }^{29}$

Es kann zu Verknüpfungen zwischen unterschiedlichen Kommunikationen kommen - etwa über den technischen Fortschritt und über die Rechtsdogmatik in Deutschland und Frankreich, welche Josef Kohler beschäftigten. ${ }^{30}$ »Konversationskreise ${ }^{3 \mathbf{I}}$ können interagieren oder interferieren, ${ }^{32}$ wenn sie in »Direktkontakt $[\mathrm{e}]$ « $^{33}$ gelangen. Als Bild für diese Orte der strukturellen Kopplung ${ }^{34}$ kann das chemische Laboratorium gewählt werden. Andere benutzen das eher naturnahe Bild der »ökologische[n] Nische[n] «. ${ }^{35}$

Die chemische Reaktion zwischen »Rechtsprinzip « und Rechtswirklichkeit lässt sich folgendermaßen beschreiben: Das Prinzip verändert nicht nur die übernehmende Rechtsordnung, auch das »eingetauchte « Prinzip muss sich zwangsläufig verändern. Verlässt man die chemische Analogie und verwendet die in der Transferdiskussion zumeist gezogene Analogie zur Medi-
2 I DE Jong, Mamadouh, Lalenis (2002) 283.

22 WATson (I993) 95: "the most fertile source of development «. De Jong, Mamadouh, Lalenis neigen tendenziell seiner Position zu: Dies. (2002) 283.

23 WATSON (2000).

24 Fögen, Teubner (2005).

25 vON Below (I905); dazu Fögen, Teubner (2005) 39.

26 LYOTARD (I990).
27 Fögen, Teubner (2005) 42, 44; in Anlehnung an LuHManN (I997) 266.

28 Zur Rolle des Rechts als »Reaktionsbeschleuniger « von technischem, wirtschaftlichem und gesellschaftlichem Wandel: SECKELMANN (2006) I-56.

29 KoHLER (I887) 27 I.

30 Zur Vita Kohlers: SpendeL (I983); KiesOw (2005). Zur dogmatischen Vorgehensweise

Kohlers: Seckelmann (2006b)

349-352.

3 I Hutter (I989) 90.

32 MAYNTZ (2002) 37.

33 Teubner (200I) $35 \mathrm{I}-38 \mathrm{I}$.

34 LuHMANN (I997) 266.

35 AsCHKe (2005) $28 \mathrm{f}$. 
zin, so verändert sich auch der "Spender « eines Prinzips. Eine Re-Implantation eines ursprünglich eigenen Prinzips führt oftmals zu »Immunreaktionen ${ }^{36}$ des fortentwickelten Spenders, ähnlich wie ein reifer Mann mit dem Herz eines Jugendlichen überfordert wäre.

\section{Das Destillat: Ein Rechtsprinzip}

Doch zurück zum Experiment: Das Recht "auf eine gute Verwaltung " bildet einen Oberbegriff über bestimmte Verwaltungspraktiken, die in Art. 4I der EU-Grundrechtecharta sowie in Art. II IоI des Verfassungsvertrags aufgezählt werden. Der Begriff der Good Administration entstammt den Bemühungen des europäischen Ombudsman um einen Code of Good Administrative Behaviour für die Organe der Europäischen Union, denen gegenüber die Europäische Grundrechtecharta auch ihre unmittelbare Schutzaussage entfaltet. In der Grundrechtecharta schwingen beim Begriff der Good Administration jedoch auch Assoziationen an den Begriff der Good Governance mit. Diesen hatte die Europäische Union in ihrem Weißbuch Europäisches Regieren vom 25. Juli 200 I als Terminus zur Bezeichnung einer Reihe von Mindeststandards für Regierungen und Verwaltungen eingeführt, die auf europäischer Ebene zu realisieren seien. ${ }^{37}$ Zur Aufnahme des ganzheitlich klingenden Rechts "auf eine gute Verwaltung «, wie es im deutschen Text heißt, in die Dokumente der Europäischen Union kam es aus verschiedenen Bestrebungen in den Jahren I999-200I, die nur kurz umrissen werden sollen. ${ }^{38}$ Diese Bestrebungen verfolgten alle das Ziel, die Identifikation der Unionsbürger mit der Europäischen Union zu verbessern. Die unter den Begriff der Good Governance zu fassenden Mindeststan- dards stellen aber keinesfalls vollständig neue Regelungen dar, sondern kodifizieren teilweise auch die Rechtsprechung des EuGH, der aus einer Gesamtschau der genannten nationalstaatlichen Bestimmungen gewisse Verfahrensgrundrechte entwickelte. ${ }^{39}$

Unmittelbarer Anlass für die Einführung des Begriffs der Good Governance als Oberbegriff für eine Gesamtheit von Mindeststandards im Weißbuch Europäisches Regieren war bereits ein früheres »Nein « der Bürger eines Mitgliedstaats gewesen. Die Bürger der Republik Irland, die wie wenige andere zuvor von der Europäischen Union profitiert hatten, hatten dem Vertrag von Nizza ihre Zustimmung verweigert. Durch den negativen Bürgerentscheid war »die Kluft zwischen der Europäischen Union und ihren Bürgern « den Mitgliedern der Europäischen Kommission offensichtlich geworden. ${ }^{\circ}$ Die Kommission hatte daher nach Maßnahmen gesucht, um »die politische Entscheidungsfindung zu öffnen, und mehr Menschen und Organisationen in die Gestaltung und Durchführung der EU-Politik einzubinden «. $^{4 \text { I }}$

Ein Grund für die Ablehnung des Vertrags von Nizza war ein Vertrauensverlust in die Unbestechlichkeit und Bürgerfreundlichkeit der Europäischen Organe gewesen, der sich auf Vorkommnisse stützte, die auch zum Rücktritt der von Jacques Santer geleiteten Europäischen Kommission im Jahre 1999 geführt hatten. Der Europäische Ombudsman, ${ }^{42}$ Jacob Söderman, erarbeitete daher Vorschläge für einen Code of Good Administrative Behaviour für die Institutionen der Europäischen Union, um diesen Einrichtungen die Anonymität zu nehmen und den Bürger für das empfundene Legitimationsdefizit der Europäischen Union zu kompensieren. ${ }^{43}$ Vergleichbare Bestrebungen bestanden auch in den Mitgliedstaaten: So führten etwa Belgien

\footnotetext{
$36 \mathrm{Zu}$ den organologischen Metaphern: KAHN-FrEUND (I978) 294-3I9.

37 Kommission der Europäischen Gemeinschaften (200I).

38 Dazu: Seckelmann (2006a).

39 Zur Entwicklung von formellrechtlichen Verfahrensgrundsätzen und solchen, die aus materiellrechtlichen Prinzipien

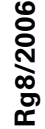
entwickelt wurden: НАІвАСН

(I998) 456; WeBER (I999) 37; sowie GorNIG, TRÜE (2000).

40 Kommission der Europäischen Gemeinschaften (200I) 3 .

4I Kommission der Europäischen Gemeinschaften (200I) 4.

42 Guckelberger (2004).

43 Europäischer Ombudsmann, Kodex für gute Verwaltungspraxis (I999). 
und die Niederlande bereits Kodizes für eine »ordnungsgemäße Verwaltung « $4 \mathbf{4}$ ein.

Daneben wurde aber auch eine Angleichung der Verwaltungsverfahren auf europäischer Ebene - und damit letztlich auch des Verwaltungsrechts der Mitgliedstaaten der Union - angestrebt. Derzeit ist eine task force der Europäischen Mitgliedstaaten mit der Frage der Angleichung ihrer Verwaltungskodizes und der Frage nach der Festlegung von Standards für die Verwaltungsverfahren der EU-Mitgliedstaaten beschäftigt.

Södermans Initiative wurde durch die Erweiterung der Europäischen Union zum I. Mai 2004 beschleunigt. Während die Europäischen Gemeinschaften der Vollzugsorganisation ihrer Mitgliedstaaten zunächst eher indifferent gegenüberstanden, stellte sich im Rahmen der Osterweiterung die Frage nach dem acquis communautaire. ${ }^{45} \mathrm{Im}$ Gefolge von Evaluationen der postsozialistischen Verwaltungen wurden Modernisierungsanforderungen formuliert, welche die Verwaltungsorganisation (insbesondere die Gewaltenteilung und die Schaffung von Überprüfungs- und Regressmöglichkeiten), die Verfahrensweisen, die Koordinationsmechanismen, die Auswahl und Organisation des Öffentlichen Dienstes und die Haushaltskontrolle der Beitrittskandidaten betrafen. Daraus ergab sich jedoch die Frage, ob die Mitgliedstaaten der Europäischen Union zwingend über einen Mindestbestand »europäischer " institutioneller Regimes verfügen sollten. Als Oberbegriff für diesen Mindestbestand etablierte sich der bereits in der Diskussion befindliche Begriff der Good Governance, der keinesfalls nur auf die Beitrittskandidaten selbst bezogen wurde, sondern auch die Verfasstheit der Mitgliedstaaten der Europäischen Union, den acquis communautaire, selbst in Frage stellte.
Der Destillationsvorgang: Von einer

Strukturauflage zum Rechtsprinzip

Der Begriff der Good Governance entstammt der Entwicklungszusammenarbeit. Anknüpfungspunkt für die Governance-Diskussion - und vor allem ein Grund für die enorme Karriere dieses Begriffs - ist die Veränderung der Rechtswirklichkeit, die gelegentlich mit dem Schlagwort »Steuerungsgegenstand veränderte Welt « zusammengefasst wird. ${ }^{46}$ Die Diskussion um Governance und Good Governance nahm ihren Ausgang in einer Zeit, in der die Entwicklungshilfe zunehmend in den Rahmen von Strukturanpassungsprogrammen eingebunden wurde. ${ }^{47}$

In den Jahren nach dem Zweiten Weltkrieg hatten die Kolonialmächte die Staaten in Afrika, Asien und Mittel- und Südamerika in die Unabhängigkeit entlassen. Letztere waren - je nach Kolonialmacht in unterschiedlicher Intensität von Europa aus verwaltet worden. Nun galt es in diesen Staaten, eine eigene Verwaltung und eine eigene Infrastruktur aufzubauen. Entwicklungshilfe wurde gezahlt, um diese Prozesse zu unterstützen. Während diese Gelder zu Zeiten des Kalten Krieges zunächst jedoch eher in einer Art Systemwettbewerb geleistet wurden, wurde seit den frühen achtziger Jahren des 20. Jahrhunderts die Ressourcenknappheit zu einem zentralen Thema der Entwicklungshilfe. Zunehmend wurde die Frage nach der Effizenz der Entwicklungsprogramme gestellt. Als Motoren dieser Debatte erwiesen sich die Weltbank und der Internationale Währungsfonds.

Um die Mängel der staatlichen Entwicklungsstrategien abzubauen, welche die Länder immer weiter in die staatliche Verschuldung trieben, sollte die weitere Vergabe von Entwicklungshilfe - abgesehen von der Unterstützung in

44 LAIS (2000) 447-482, SCHILLING (2000) 3 .

45 KöNIG (2003) 53. Zum OECD-

Programm Support for Improve-

ment in Governance and Manage-

ment in Central and East Euro-

pean Countries, vgl. SPEer (200I).

46 Hill (2005).

47 KÖNIG (2002) I7; THEOBALD

(200Ia); THEOBALD (200IC);

FUSTER (I998); ADAM (200I). 
aktuellen Notlagen - von der Umsetzung struktureller Reformen abhängig gemacht werden. Diese Reformen sollten den Markt fördern und den Rückzug des Staates aus den wirtschaftlichen Aktivitäten vorantreiben. ${ }^{48}$ Als sich infolge der ersten Programme ein reines Liberalisierungsprogramm als nur teilweise effizient erwies, wurde die Rolle des Staats als Verteilungs- und Modernisierungsagentur wiederentdeckt. ${ }^{49}$ Die Rolle der institutionellen Regimes, der gesetzlichen oder organisatorischen Regeln für das effiziente Funktionieren von Märkten, rückte zunehmend in den Blick. ${ }^{5 \circ}$

In einer Studie der Weltbank aus dem Jahre I 989 über Vergabedingungen von Geldern für das südliche Afrika wurden daher insbesondere die institutionellen Rahmenbedingungen für die Funktionsfähigkeit von Märkten betont. Governance wurde von der Weltbank in einem Epoche machenden Bericht über das südliche Afrika als "the exercise of political power to manage a nation's affairs " definiert, ${ }^{5 \mathrm{I}}$ also als "Art und Weise, in welcher bei dem Management der wirtschaftlichen und sozialen Ressourcen eines Landes im Interesse einer nachhaltigen Entwicklung die Ausübung von Macht gehandhabt wird « ${ }^{52}$ Die Weltbank, der ihrem Statut nach eine politische Aktivität ausdrücklich untersagt ist, ${ }^{53}$ begann über diesen Begriff zumindest Kriterien an die Effizienz und Transparenz der Verfasstheit eines Staates und seiner Organisationen zu stellen.

Die Weltbank verknüpfte mit ihrer Definition der Good Governance als »Fähigkeit, auf auftretende Probleme angemessen zu reagieren ", formelle und materielle Kriterien - wie die Respektierung der Menschenwürde der Bürger und die Verantwortlichkeit der Regierungsführung. ${ }^{54}$ Im Zentrum der Betrachtung standen nicht mehr nur der Staat, sondern auch seine Umweltbe- ziehungen. Nicht einzig formelles Verwaltungshandeln, sondern gerade auch das informelle Zusammenwirken des Staates mit zivilgesellschaftlichen Akteuren wurde nach dem (Good) Governance-Konzept einer Effizienzbetrachtung unterzogen, die weniger nach den Rechtmäßigkeitsanforderungen für das Verfahren fragte als nach den Ergebnissen staatlichen Handelns.

Der Good Governance-Ansatz ist daher zugleich umfangreicher wie enger als der des deutschen Rechtsstaatsprinzips. Er meint über eine willkürfreie Verwaltung hinaus auch eine solche, welche bürgerfreundlich ist und auf neue Probleme angemessen reagieren kann. Diese Umstellung von einer Ergebnis-Rechtmäßigkeit auf eine Verfahrensrichtigkeit und ein die Schaffung von Strukturen zur Problemlösung ausgerichtetes Konzept $^{55}$ hat vor allem angloamerikanische Wurzeln, insbesondere die beschriebenen Rechtsprinzipien des common law, die in vielen ehemaligen Kolonien ohnehin weitergalten. Im Blickpunkt dieses ganzheitlichen Konzepts steht nicht mehr nur der Staat, sondern die Gesamtheit der Akteure, welche sich um das Gemeinwohl kümmern. Die Aufgabe der Fixierung der Regelungsaussage auf den Staat schürt auf der anderen Seite jedoch die Befürchtung, dass der Begriff der Good Governance das Rechtsstaatsprinzip, um im Bild der chemischen Reaktion zu bleiben, aufweichen kann. Neben Aussagen, die noch dem hard law zugerechnet werden können, wie die Existenz der Prozessgrundrechte, enthält er bislang - auch wenig justiziable Elemente wie die »Bürgerfreundlichkeit «, die bislang nur in Großbritannien flächendeckend gemessen und evaluiert wird. ${ }^{56}$

\footnotetext{
48 Murphy (2002) 2.

49 NyANG'ORo (I999).

50 North (I988); Theobald

(200Ib); EVANS, RUESCHEMEYER, SKOCPOL (I999).

5 I WORLD BANK (I989) 60.

52 Übersetzung von Klages in: Ders. (2000) 3 .

53 Diese Restriktion ergibt sich aus

Artikel ro des Weltbankstatuts

(http://worldbank.org); dazu:

ஜั

ADAM (2000) 272.

54 World Bank (1989) 3; dazu: ADAM

(2000) 272.

55 Hoffmann-Riem (2004) 25 f.;

BULLINGER (2002) 25.

56 Dazu: Bovaird, LÖFfler (2003). 


\section{Zur Eigendynamik von Destillaten}

Rechtsprinzipien haben, wie Josef Kohler schrieb, die Eigenschaft, bei der Reaktion mit der Rechtswirklichkeit von (fremden) Rechtsordnungen eine eigene Dynamik zu entwickeln. Der Begriff der "guten Gouvernanz « 57 unterwarf daher schließlich auch die Organisation der »entwickelten ${ }^{58}{ }^{8}$ Staaten einem gesteigerten Rechtfertigungsbedürfnis. Der Begründungsaufwand für Defizite innerhalb der demokratischen Struktur dieser Staaten, insbesondere der für das bekannte Demokratiedefizit der Europäischen Union, erhöhte sich angesichts derjenigen Erwartungen, die man an die Empfängerländer von Entwicklungshilfe wie - unter anderen Vorzeichen - für die Beitrittskandidaten zur Europäischen Union formulierte. Von einem Oberbegriff über die von einer Regierung zu gewährleistenden Mindeststandards für die Teilnahme an internationalen Strukturentwicklungsprogrammen avancierte (Good) Governance nach und nach zu einem »Kernbegriff der zeitgenössischen politischen Ökonomie «.59 Der Terminus wurde »zu einem Leitbegriff aktueller Erörterungen über die Aufgaben moderner Staatswesen und über die Wege«, die "ganz allgemein zu der zukunftshaltigen Weiterentwicklung institutioneller Strukturen hinführen ${ }^{6}{ }^{60}$

In Großbritannien wurde die Stärkung der Problemlösungsfähigkeit des Staates im Zeichen sinkender staatlicher Einnahmen unter dem Begriff der Governance etwa durch das Regierungskonzept Modernising Government der Regierung Blair eingeführt und in Deutschland seitdem intensiv rezipiert. ${ }^{6 \mathbf{I}}$
Vorsicht explosiv! Folgen für das deutsche Rechtsstaatsprinzip?

Was nun bedeutet die Aufnahme eines für das deutsche Rechtsstaatsverständnis recht offenen und dynamischen Begriffs in die Grundrechtecharta der Europäischen Union und in den Europäischen Verfassungsvertrag? Es ist zu konzedieren, dass durch die Bildung von Regelbeispielen der Begriff der Good Administration deutlich schärfere Konturen aufweist als derjenige der Good Governance. Dennoch tun sich Staaten mit etatistischer Tradition wie Deutschland oder Frankreich, die eine starke Trennung von Justiz und Verwaltung vornehmen, schwer mit der Beschreibung von inhaltlichen Qualitätsvorgaben für die drei Gewalten, die über die Bindung an die Verfassung sowie Recht und Gesetz hinausgehen. Daher wurde - insbesondere von französischer und deutscher Seite - oftmals der Wunsch nach einer weiteren Konkretisierung beider Begriffe erhoben, die im Endeffekt auf die Aufgabe der Qualitätsbeschreibung "gut « in der "guten Governanz« hinausläuft. ${ }^{62}$ Die Aufgabe des Begriffs "gut « jedoch hat teilweise nur terminologischen Charakter. Auch der scheinbar neutrale Begriff der Governance ${ }^{63}$ ist nicht unbedingt inhaltsneutral, sondern oftmals inhaltlich mit einer politischen Vision, nämlich derjenigen nach einer - globalen ${ }^{64}$ - Zivilgesellschaft ${ }^{65}$ oder dem Wunsch nach einer Art transdisziplinärer lingua franca ${ }^{66}$ verbunden.

Eine chemische Reaktion - verstärkt durch die katalytische Wirkung eines Rechtsprinzips -, wie sie Josef Kohler beschrieb, könnte auch deswegen im deutschen Recht erfolgen, als der Rechtscharakter der EU-Grundrechte, welche über ihre qualitative Aufladung auch auf außerrechtliche Perspektiven ${ }^{67}$ verweisen, eher vage ist. Die EU-Kommission verfügt grundsätzlich

\footnotetext{
57 KÖNIG (2002) I7.

58 KLAGES (2000) 3.

59 MURPHY (2002) I.

60 KLAGES (2000) 3. Werner Jann spricht anschaulich von einem "Modebegriff «: JANN (2002) 302; zu diesem Komplex auch: Ders. (2005) 2I-43.

6I Dazu beispielhaft JanN (2005); Hill (2005); Klenk, Nullmeier (2002).

62 Etwa von: Demers (I997).
}

63 Neben dem Begriff der (Public) Good Governance wird teilweise auch der Terminus Modern Governance verwendet: KUHLMANN (2004) 22I-24O.

64 STICHWEH (2000) 25.

65 Teubner (2004) 7I-87.

66 Trute, Denkhaus, Kühlers (2004) 45I.

67 BAer (2004) 225; Vosskuhle (200I). 
über keine Kompetenz für Vorschriften zur Gestaltung von Verwaltungsverfahren. Eine »Durchsteuerung « der Verwaltungsorganisation stünde daher - zumindest, wenn sie nicht durch den Verfassungsvertrag abgesichert wäre, sondern nur mittelbar durch die grundsätzlich nur deklaratorische EU-Grundrechtecharta erfolgen sollte - in Widerspruch zum Prinzip der begrenzten Einzelermächtigung. ${ }^{68}$ Für die Möglichkeit der "Zündung « einer solchen Reaktion spricht, dass die EU-Kommission bereits zuvor mit so genannten »Querschnittsklauseln «, insbesondere im Bereich der Dienstleistungen, ${ }^{69}$ operiert hat, um auf die nationalstaatlichen Verwaltungsverfahren einzuwirken. Der EuGH wird zu dieser Frage Stellung nehmen müssen. Seine bisherige Rechtsprechung zur Kollision der Grundfreiheiten mit den nationalen Grundrechten ${ }^{70}$ deutet jedoch darauf hin, dass er den nationalen Grundrechten und anderen nationalen Prinzipien von Verfassungsrang nicht unbedingt einen Vorrang vor der EU-Grundrechtecharta einräumen wird.

Unter Laborbedingungen?

Recht und Sozialer Wandel

Die Beobachtung der aktuellen Diskussion um den EU-Verfassungsvertrag und die EUGrundrechtecharta zeigt mithin, dass es bei der Begegnung von Rechtsprinzipien und Rechtswirklichkeit $\mathrm{zu}$ unintendierten Nebeneffekten kommen kann. Irritationen sind unvermeidlich, sei es, weil die Wirkungen der Reaktion nicht vorher bekannt waren, sei es, dass weitere Intermittenten auftraten, etwa weil das Reagenzglas noch Rückstände aufwies. Die Begriffe Rechtstransfer - und auch das extremere, weil organologische Wort legal transplant - sind da- her als Metaphern für situative Irritationen zwischen verschiedenen Systemen anzusehen. Hinter den ontologisch aufgeladenen Bezeichnungen verbirgt sich die Frage nach der Bewältigung sozialen Wandels durch Recht. Gunther Teubner hat diese Beobachtung anhand einer Analyse der Reaktion der britischen Rechtsprechung auf das Prinzip Treu und Glauben (good faith) der EU-Verbraucherschutzrichtlinie vorgenommen. Seine Prognose lautete, dass die britische Interpretation von good faith niemals zu der unglaublichen Ausdifferenziertheit und - durchaus ambivalent zu betrachtenden - abstrakten Dogmatisierung führen werde, welche die Kommentierung des $\mathbb{2 4 2} \mathrm{BGB}$ in Deutschland angenommen habe. Ähnlich gestaltet sich die Situation im Verwaltungsrecht: Das deutsche Verhältnismäßigkeitsprinzip fand über die Rechtsprechung des EuGH auch Eingang in die britische Jurisdiktion und sorgt so für eine Irritation des dortigen Rechtssystems. ${ }^{7 \mathbf{I}}$

Es ist zu erwarten, dass die deutsche Rechtsprechung bei der Auslegung der Europäischen Grundrechtecharta und namentlich des Rechts auf eine gute Verwaltung versuchen wird, das Prinzip in die Systemlogik des deutschen Verfassungs- und Verwaltungsrechts zu integrieren. In ihrem Bemühen um die Konstitution von Sinn ${ }^{72}$ wird die deutsche Rechtsprechung - ähnlich wie die von Teubner beschriebene englische Jurisdiktion - eine Auslegung zu finden suchen, die mit den deutschen Grundrechten und Prinzipien von Verfassungsrang (insbesondere dem Rechtsstaats- und dem Demokratieprinzip) vereinbar sein wird. Die Irritation des deutschen Rechts wie der deutschen Gesetzgebung wird gleichwohl nicht zu unterschätzen sein, wie die nahezu serielle Anpassung und Produktion von Gesetzen zeigt, die durch Richtlinien der Europäischen Kommission oder Urteile des EuGH be-

\footnotetext{
68 RiEdEL (I999).

69 KÄMMERER (2004) 39; HATJE

(2000), Rz. 5.

$70 \mathrm{EuGH}, \mathrm{Rs} . \mathrm{C}-\mathrm{I}$ I 2/oo, Slg. 2003, I-5659 (Schmidtberger, Österreich).

7I HaRlow (2000) 240. Konkrete Beispiele für die Irritation anderer nationaler Rechtsordnungen durch die Rechtsprechung des

Chiner (2004) sowie den anderen

Länderberichten in diesem Band. 
dingt wird. Dass in Deutschland in diesen Fällen - anders als in manchen anderen Mitgliedstaaten - in der Regel der Gesetzgeber tätig werden muss, ergibt sich aus dem Vorbehalt des Gesetzes (Art. 80 Abs. I GG).

Inwieweit es zu einer "Aufweichung « des Rechtsstaatsprinzips kommen wird, ist daher schwer zu beurteilen. Prinzipiell wird seine Aussage Bestand haben müssen, wie die »Maastricht ${ }$-Entscheidung ${ }^{73}$ des Bundesverfassungsgerichts zeigt. Gleichwohl ist eine interpretatorische Arbeit der Gerichte bei der systematischen Gesamtschau dieses Prinzips mit der EU-Grundrechtecharta zu erwarten, die der Perspektive nach inhaltlich $\mathrm{zu}$ ähnlichen Ausformungen kommen wird wie das deutsche Rechtsstaatsprinzip, wenn auch vielleicht nicht in dessen Abstraktheit. Diese wird derzeit ohnehin durch die eher prätorische EuGH-Rechtsprechung herausgefordert. In der common law-Tradition wird jedoch über die Garantie von Prozessgrundrechten und anderen Verfahrensregeln pfadabhängig ein Effekt erreicht, der dem deutschen Rechtsstaatsprinzip vergleichbar ist; mehr noch, diese Regeln bildeten das Vorbild, aus dem das Rechtsstaatsprinzip abstrahiert wurde. Montesquieu, der zugleich vor einer naiven Form der Übernahme von Institutionen warnte, ${ }^{74}$ legte mit seiner Beschreibung der Demokratie in Amerika die gedanklichen Grundlagen für die Dogmatik des von ihm nicht erwähnten Grundsatzes der Gewaltenteilung $^{75}$ - und damit für einen essentiellen Bestandteil des deutschen Rechtsstaatsprinzips.

Insofern lautet die Antwort auf die Frage nach der Möglichkeit von Rechtstransfer: Eine Kommunikation zwischen Rechtsordnungen ist wünschenswert und im Zeichen der europäischen Mehrebenenpolitik auch unvermeidlich. ${ }^{76}$ Eine splendid isolation einer Rechtsord- nung ist eine lebensferne Vorstellung, die der Kommunikation über Recht in der Rechtswissenschaft auch zu der Zeit nicht gerecht wurde, als die Frage nach einer European Governance noch nicht gestellt worden war. Ein emphatisches Konzept von Rechtstransfer, von Alan Watson ebenso euphorisch vertreten wie von Pierre Legrand abgelehnt, ist jedoch etwas hoch gegriffen. Die Vorstellung einer »Episodenverknüpfung «, 77 einer »Interferenz ${ }^{48}{ }^{8}$ oder einer Überlappung von »Konversationskreisen « 79 wird dem Phänomen eher gerecht.

Sind diese Vorgänge eigentlich steuerbar? Gibt es eine Art »Laborpersonal «, das versuchen kann, Einfluss auf die Transferprozesse zu nehmen? Worin besteht etwa die Rolle der obersten Gerichtsbarkeit? Das deutsche Bundesverfassungsgericht hat in seiner »Maastricht «Entscheidung und den vorausgegangenen »Solange «-Entscheidungen den Versuch unternommen, eine Rahmensetzung für Transferprozesse vorzunehmen. Experimente benötigen unter bestimmten Voraussetzungen Initiatoren. Diese können in Kollektivakteuren wie der Rechtsprechung, internationalen Organisationen, der Europäischen Kommission oder schließlich auch den Nationalstaaten zu suchen sein. ${ }^{8 \circ}$ Die Interaktion zwischen ihnen vollzieht sich nach systemischen Kriterien, die oft mehr mit der Erwartung von Verhalten als mit diesem selbst zu tun haben. ${ }^{8 \mathbf{I}}$

Das Handeln der Kollektivakteure lässt sich mit Metaphern umschreiben - wie derjenigen von der $»$ regulierten Selbstregulierung ${ }{ }^{82}$ oder der »Regelungsstruktur «. ${ }^{83}$ Eine andere mögliche Metapher ist diejenige des Labors. Ist man sich bewusst, dass es sich um Metaphern handelt, die besonders geeignet sind, situative Kommunikationen zwischen Systemen zu veranschaulichen, so kann man sich ihrem ästhetischen Reiz

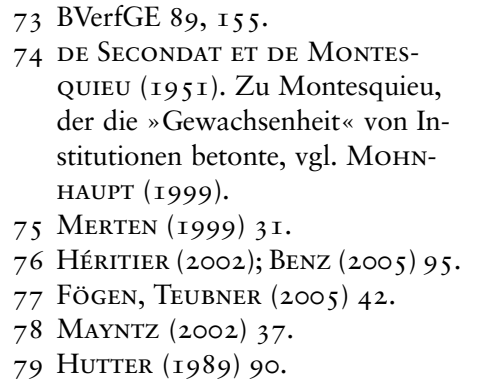

73 BVerfGE 89, I 55. der die "Gewachsenheit « von Institutionen betonte, vgl. MoHNHAUPT (I 999).

75 Merten (r999) 3I

77 Fögen, Teubner (2005) 42.

79 Hutter (I989) 90.

80 Einen bedeutenden Versuch, Organisationen und Teilsysteme als korporative Akteure mit der autopoietischen Theorie Luhmanns zu vereinen, bildet der Ansatz von Helmut Willke (I995); zu weiteren Ansätzen: BraUn (2000) 107. Die Bedeutung der Akteure nimmt gegenüber den Institutionen nach der Diagnose von ARTHur Benz in der europäischen
Mehrebenenpolitik sogar zu: DeRs. (2005) II 6.

8I Akselrod (I995).

82 Dazu die Beiträge in: Regulierte Selbstregulierung als Steuerungskonzeption des Gewährleistungsstaats, Berlin 200I (= Die Verwaltung, Beiheft 4).

83 Dazu insbesondere die Beiträge bei: SCHupPert (2005) sowie Trute, Denkhaus, Kühlers (2004); Denkhaus (2004). 
kaum entziehen. ${ }^{84}$ Daher soll es in Anlehnung an Josef Kohler heißen: Juristen sind Chemiker. Trotz der heftigen Irritationen der politischen Systeme durch die Ablehnung des EU-Verfassungsvertrags werden Rechtsprechung und Rechtsdogmatik nunmehr beginnen, die EUGrundrechtecharta mit den eigenen Verfassungs- verbürgungen zu vergleichen. Sie werden ganz im Sinne Kohlers mithin neue "Combinationen " suchen, aus welchen »neue fruchtbare Gebilde ${ }^{85}$ hervorgehen können.

\section{Margrit Seckelmann}

\section{Literatur}

M. AdAm (2000), Die Entstehung des Governance-Konzepts bei Weltbank und UN. Die EZ wird politischer, in: Entwicklung und Zusammenarbeit 4I, 272-274.

M. ADAM (200I), Governance als Ansatz der Vereinten Nationen. Das Beispiel des UNDP, in: Governance als entwicklungspolitischer Ansatz, hg. von K. KöNIG u. DEMS., Speyer, I I-33.

R. Akselrod (I995), Die Evolution der Kooperation, München.

M. AschKe (2005), Einheit. Theoretische Aspekte des Großtransfers von Recht und juristischem Personal, in: Rechtsgeschichte 7 , I3-32.

S. BAER (2004), Schlüsselbegriffe, Typen und Leitbilder als Erkenntnismittel und ihr Verhältnis zur Rechtsdogmatik, in: Methoden der Verwaltungsrechtswissenschaft, hg. von E. SchmidT-AssMANN u. W. HofFMANN-Riem, Baden-Baden, 223-25I.

O. BäHR ([I864] I96I), Der Rechtsstaat. Eine publicistische Skizze, Nachdruck, Aalen.
G. von Below (1905), Die Ursachen der Rezeption des römischen Rechts in Deutschland, München, Berlin.

W. Benjamin (I977), Die Aufgabe des Übersetzers, in: Ders., Illuminationen. Ausgewählte Schriften, Frankfurt a. M., 50-62.

A. Benz (200I), Der moderne Staat. Grundlagen der politologischen Analyse, München, Wien.

A. Benz (2005), Governance in Mehrebenensystemen, in: GovernanceForschung. Vergewisserung über Stand und Entwicklungslinien, hg. von G. F. SCHUppert, BadenBaden, 95-I 20.

C. BöHRET, G. KONZENDORF (I997), Ko-Evolution von Gesellschaft und funktionalem Staat, Opladen.

T. Bovaird, E. Löffler (2003), Public Management und Governance, London.

D. Braun (2000), Politische Steuerung als systemtheoretisches Problem, in: Politische Steuerung zwischen System und Akteur. Eine Einführung, hg. von S. LANGE u. DEMS., Opladen, 99-I76.
M. Bullinger (2002), Das Recht auf gute Verwaltung nach der Grundrechtecharta der EU, in: Der Wandel des Staates vor den Aufgaben der Gegenwart, hg. von C.-E. Eberle u. a., München.

R. COASE (I960), The Problem of Social Cost, in: Journal of Law and Economics 3, I-44.

M. Demers (I997), La gouvernance de la gouvernance: Faut-il freiner l'engouement?, in: Gouvernance: Concepts et Applications/Governance: Concepts and Applications, hg. von J. CORKERY, Brüssel, 367-380.

W. Denkhaus (2004), Neue Institutionenökonomik und das Governancekonzept - Zum Wandel der ökonomischen Theorie und ihren Implikationen für die Verwaltungswissenschaft, in: Recht und Ökonomik, hg. von M. BungeNBERG u. a., München, 33-60.

M. DE Jong, V. Mamadouh, K. LALENIS (2002), Drawing Lessons about Lesson Drawing. What the case reports tell us about institutional transplantations, in: Dies.,

84 So wird auch der Hinweis auf die verschiedenen Möglichkeiten zur Schließung diskursiver Operationen von Fögen u. TeubneR verstanden: Dies. (2005) 44.

85 Kohler (I887) $27 \mathrm{I}$. 
Institutional Transplantation, Dordrecht, 283-299.

B. Dutzler, A. LuKaschek, E. VRANES, B. WeIdel (200I), Grundrechte für Europa. Die Europäische Union nach Nizza, in: Zeitschrift für Verwaltung, I78-I83.

Europäischer Ombudsman, Kodex für gute Verwaltungspraxis vom 28. Juli 1999, Download unter: http://www.euro-ombudsman.eu. int/recommen/pdf/de/codeI_de.pdf (einges. am 8.I 2.2005).

P. B. Evans, D. Rueschemeyer, T. SKocpol (Hg.) (r 999), Bringing the State back in, Cambridge.

M. Th. Fögen, G. Teubner (2005), Rechtstransfer, in: Rechtsgeschichte $7,38-45$.

TH. Fuster (I998), Die »Good Governance «-Diskussion der Jahre I 989 bis I994, Stuttgart.

R. von GNeIst (I 879), Der Rechtsstaat und die Verwaltungsgerichte, 2. Auflage, Berlin.

G. Gornig u. Ch. Trüe (2000), Die Rechtsprechung des EuGH und des EuG zum Europäischen Verwaltungsrecht, Teil $\mathrm{I}_{-3}$, in: Juristenzeitung, 395-406; 446-457; $50 \mathrm{I}-5 \mathrm{I} 2$.

A. GuCKelberger (2004), Der Europäische Bürgerbeauftragte und die Petitionen zum Europäischen Parlament - eine Bestandsaufnahme zu Beginn des 2I. Jahrhunderts, Berlin.

C. HARLOW (2000), Export. Import. The Ebb and Flow of English Public Law, in: Public Law, 240-253.

G. НаiвACH (I998), Die Rechtsprechung des EuGH zu den Grundsätzen des Verwaltungsverfahrens, in: Neue Zeitschrift für Verwaltungsrecht, 456-462.

A. Hatje (2000), Kommentierung von Art. I6, in: EU-Kommentar, hg. von J. Schwarze, Baden-Baden.

A. Héritier (2002), New Modes of Governance in Europe: PolicyMaking without Legislating?, in: Common Goods: Reinventing European and International Governance, hg. von DERs., Lanham, Md., I 85-206.

H. Hill (2005), Good Governance Konzepte und Kontexte, in: Governance-Forschung. Vergewisserung über Stand und Entwicklungslinien, hg. von G.F. SChuppert, Baden-Baden, 220-250.
H. HiLl (2005), Governance als Reformstrategie - Vom Wandel und der Bedeutung verwaltungspolitischer Leitbilder, in: GovernanceForschung. Vergewisserung über Stand und Entwicklungslinien, hg. von G. F. SCHuppert, BadenBaden, $2 \mathrm{I}-43$.

W. Hoffmann-Riem (2004), Methoden einer anwendungsorientierten Verwaltungsrechtswissenschaft, in: Methoden der Verwaltungsrechtswissenschaft, hg. von E. Schmidt-Assmann u. Dems., Baden-Baden, 9-72.

M. Hutter (I989), Die Produktion von Recht. Eine selbstreferentielle Theorie der Wirtschaft, angewandt auf den Fall des Arzneimittelpatentrechts, Tübingen.

A. Iber (2002), Der Status der Grundrechtscharta im Gemeinschaftsrecht: Derzeitige Verbindlichkeit und Zukunftsperspektiven der Charta - insbesondere im Verfassungskonvent, in: Zeitschrift für Europarechtliche Studien 5, 483505 .

W. JANN (2002), Wandel verwaltungspolitischer Leitbilder: Vom Management zur Governance, in: Deutsche Verwaltung an der Wende zum 2I. Jahrhundert, hg. von K. KöNIG, Baden-Baden, 279-304.

J. A. KäMMERER (2004), Strategien zur Daseinsvorsorge. Dienste im allgemeinen Interesse nach der "Altmark «-Entscheidung des EuGH, in: Neue Zeitschrift für Verwaltungsrecht, 28-34.

O. Kahn-Freund (I978), On Uses and Misuses in Comparative Law. Selected Writings, London, 294319.

R. M. KIEsOw (2005), Josef Kohlers Poesie, in: Summa. Dieter Simon zum 70. Geburtstag, hg. von Dems., R. Ogorek, S. Simitis, Frankfurt am Main, 297-3 I 8.

H. Klages (2000), Good Governance in entwickelten Ländern? Erfordernisse und Handlungsspielräume "aktivierender « Politik, in: Good Governance und Qualitätsmanagement - Europäische und internationale Entwicklungen, hg. von H. Hill u. DEMs., Speyer.

T. Klenk, F. Nullmeier (2003), Public Governance als Reformstrategie, Düsseldorf.
K. KÖNIG (2002), Gute Gouvernanz als Steuerungs- und Werkkonzept des modernen Verwaltungsstaates, in: Verwaltete Regierung. Studien zur Regierungslehre, hg. von DERs., Köln u. a., I I-37.

K. KöNIG (2003), Governance im Mehrebenensystem, in: Aktuelle Fragen zu Verfassung und Verwaltung im europäischen Mehrebenensystem, hg. von K.-P. Sommermann, 2. Auflage, Speyer, 4570.

J. KoHLER (I 887), Die schöpferische Kraft der Jurisprudenz, in: Jahrbücher für die Dogmatik des heutigen römischen und deutschen Privatrechts 25 (N.F. 8), 262-297.

Kommission der Europäischen Gemeinschaften, Europäisches Regieren. Ein Weißbuch, Brüssel den 25.7.200I (KOM 200I) 428 endgültig, 3 (download unter http://europa.eu.int/eur-lex/lex/ LexUriServ/site/de/com/200I/ com200I_0428deor.pdf, einges. am 8.I2.2005).

S. Kuhlmann (2004), Kooperativer Gesetzesvollzug in ostdeutschen Kommunen: Vom Systembruch zu "Modern Governance «?, in: Rechtssoziologie 25, 22I-240.

M. LaIs (2000), Das Recht auf eine gute Verwaltung unter besonderer Berücksichtigung der Rechtsprechung des Europäischen Gerichtshofs, in: Zeitschrift für Europarechtliche Studien 5, 447-482.

P. LEgrand (I997), The Impossibility of Legal Transplants, in: Maastricht Journal of European and Comparative Law 4, I I I-I 24.

N. LuHMANN (I987), Soziale Systeme. Grundriß einer allgemeinen Theorie, Frankfurt a. M.

N. Luhmann (I997), Das Recht der Gesellschaft, 2. Auflage, Frankfurt a. M.

F. Lyotard (I990), Das postmoderne Wissen, Wien.

S. Magiera (2003), Die Bedeutung der Grundrechtecharta für die Europäische Verfassungsordnung, in: Europäische Verfassungsordnung, hg. von D. H. Scheuing, BadenBaden, II $7-\mathrm{I}_{3} 2$.

M. Mahlmann (2000), Die Grundrechtscharta der Europäischen Union, in: Zeitschrift für Europarechtliche Studien 3, 4I9-444.

R. MAYNTZ (2002), Zur Theoriefähigkeit makro-sozialer Analysen, in: 
Akteure - Mechanismen - Modelle. Zur Theoriefähigkeit makro-sozialer Analysen, hg. von DERS., Frankfurt, New York, 7-43.

D. Merten (I999), Montesquieus Gewaltenteilungslehre und deutsche Verfassungsstaatlichkeit, in: Montesquieu. 250 Jahre »Geist der Gesetze «: Beiträge aus Politischer Wissenschaft, Jurisprudenz und Romanistik, hg. von P.-L. Weinacht, Baden-Baden, $3 \mathrm{I}-62$.

R. von MoHL (I 832), Die PolizeiWissenschaft nach den Grundlagen des Rechtsstaates, Band I, Tübingen.

H. Mohnhaupt (I999), Deutsche Übersetzungen von Montesquieus "De l'esprit des lois «, in: Montesquieu. 250 Jahre "Geist der Gesetze «: Beiträge aus Politischer Wissenschaft, Jurisprudenz und Romanistik, hg. von P.-L. WeINACHT, Baden-Baden, I 35-I 5 I.

M. J. Montoro Chiner (2004), Europäisierung des Verwaltungsverfahrensrechts. Länderbericht Spanien, in: Europäisches Verwaltungsverfahrensrecht, hg. von H. Hill, R. Pitschas, Berlin, I27-I 53 .

E. C. Murphy (2002), Good Governance - Ein universal anwendbares Konzept?, in: Internationale Politik 8, I-9.

D. C. NorTH (I988), Theorie des institutionellen Wandels. Eine neue Sicht der Wirtschaftsgeschichte, Tübingen.

J. E. Nyang'oro (I999), Hemmed in? The State in Africa and Global Liberalization, in: States and Sovereignty in the Global Economy, hg. von D. SMITH, D. Solinger, S. TopIK, London, 264-277.

M. PrÖHL (Hg.) (2002), Good Governance für Lebensqualität vor Ort. Internationale Praxisbeispiele für Kommunen, Gütersloh, I6-I I 5.

Regulierte Selbstregulierung als Steuerungskonzeption des Gewährleistungsstaats, in: Ergebnisse des Symposiums aus Anlaß des 60. Geburtstages von Wolfgang Hoffmann-Riem, Berlin 200I (= Die Verwaltung, Beiheft 4).

E. Riedel (I999), Rechtliche Optimierungsgebote oder Rahmensetzungen für das Verwaltungshandeln?, in: Veröffentlichungen der Ver- einigung der Deutschen Staatsrechtslehrer 58, I77-2I7.

M. RufFert (2004), Die Methodik der Verwaltungsrechtswissenschaft in anderen Ländern der Europäischen Union, in: Methoden der Verwaltungsrechtswissenschaft, hg. von E. SCHMidT-Assmann, W. Hoffmann-RIEM, BadenBaden, I65-22I.

TH. SCHilling (2000), Bestand und allgemeine Lehren der bürgerschützenden allgemeinen Rechtsgrundsätze des Gemeinschaftsrechts, in: Europäische Grundrechte-Zeitschrift, 3-43.

W. Schroeder (I998), Demokratie, Transparenz und die Regierungskonferenz - Überlegungen zur Legitimität der Europäischen Union, Kritische Vierteljahresschrift für Gesetzgebung und Rechtswissenschaft 8I, 423-440.

G. F. SChuppert (Hg.) (2005), Governance-Forschung, Vergewisserung über Stand und Entwicklungslinien, Baden-Baden 2005.

M. Seckelmann (2006a), Good Governance - Importe und Re-Importe, in: Rechtstransfer, hg. von V. Duss, N. Linder u. a., München.

M. Seckelmann (2006b), Industrialisierung, Internationalisierung und Patentrecht im Deutschen Reich, I87I-I9I4, Frankfurt a. M.

Ch. de Secondat et de MontesQuiEU (I95I), Vom Geist der Gesetze, in neuer Übertragung eingel. und hg. von E. Forsthoff, Band I, Tübingen.

K.-P. SommermanN (2002), Verwaltung im Rechtsstaat, in: Deutsche Verwaltung an der Wende zum 2I. Jahrhundert, hg. von K. KöNIG, Baden-Baden, 97-I I 8.

B. SPEER (200I), Das SIGMA-Progamm der OECD: Ein Governance-Ansatz für Mittel- und Osteuropa?, in: Governance als entwicklungspolitischer Ansatz, hg. von K. KöNIG, M. ADAM, Speyer, 67-89.

G. SPENDEL (I983), Josef Kohler. Bild eines Universaljuristen, Heidelberg.

R. StichweH (2000), Die Weltgesellschaft. Soziologische Analysen, Frankfurt am Main.

M. STOLLEIS (I992), Geschichte des öffentlichen Rechts in Deutschland, Zweiter Band: I800-I9I4, München.
P. J. Tettinger (200I), Die Charta der Grundrechte der Europäischen Union, in: Neue Juristische Wochenschrift, IOIO-IOI 5 .

G. TeubNer (200I), Rechtsirritationen: Zur Koevolution von Rechtsnormen und Produktionsregimes, in: Moral und Recht im Diskurs der Moderne: Zur Legitimation gesellschaftlicher Ordnung, hg. von G. Dux, F. Welz, Opladen, 35I$38 \mathrm{I}$.

G. Teubner (2004), Global Private Regimes: Neo-Spontaneous Law and Dual Constitution of Autonomous Sectors?, in: Public Governance in the Age of Globalization, hg. von K.-H. LADEUR, Aldershot, 7 I-87.

Ch. Theobald (200Ia), Governance in der Perzeption der Weltbank, in: Governance als entwicklungs- und transformationspolitisches Konzept, hg. von K. KöNIG, München, 55-I 27.

Ch. Theobald (200Ib), Zehn Eckpunkte zu Good Governance, in: Governance als entwicklungspolitischer Ansatz, hg. von K. KöNIG, M. AdAM, Speyer, 35-65.

Ch. Theobald (200Ic), Zur Ökonomik des Staates: Good Governance und die Perzeption der Weltbank, Baden-Baden.

H.-H. Trute, W. Denkhaus, D. KühLERS (2004), Governance in der Verwaltungsrechtswissenschaft, in: Die Verwaltung 37, $45 \mathrm{I}-473$.

A. Vosskuhle (200I), »Schlüsselbegriffe « der Verwaltungsreform Eine kritische Bestandsaufnahme, in: Verwaltungsarchiv 92, I842 I 5 .

A. Watson (1993), Legal Transplants. An Approach to Comparative Law, 2nd Edition, Athens, London.

A. Watson (2000), Legal Transplants and European Private Law, in: Electronic Journal of Comparative Law 4.4, http://www.ejcl.org/44/ art $44^{-2}$.html (einges. am 8.I2.2005).

A. Weber (1999), Entwicklungen im europäischen Verwaltungsrecht, in: Öffentliche Verwaltung in Europa, hg. von J. IPSEN, B. STÜER, Köln u. a., 35-5 I.

H. WILLKe (I995), Systemtheorie III: Steuerungstheorie. Grundzüge einer Theorie der Steuerung komplexer Sozialsysteme, Stuttgart, Jena. 
J. WOLF (2002), Vom Grundrechtsschutz » in Europa « zu allgemeinverbindlich geltenden europäischen Grundrechten - Wege der Grundrechtssicherung unterhalb der Ebene europäischer Verfassungsgebung, in: Der Grund- rechtsschutz in Europa, hg. von J. BRÖHMER, Baden-Baden, 9-65. World Bank (1989), Sub-Saharan Africa: From Crisis to Sustainable Growth. A Long-Term Perspective Study, Washington.

J. Ziekow (2002), Die Funktion des Allgemeinen Verwaltungsrechts bei der Modernisierung und Internationalisierung des Staates, in: Internationalisierung von Staat und Verfassung im Spiegel des deutschen und japanischen Staatsund Verwaltungsrechts, hg. von R. Pitschas, S. Kisa, Berlin, I87$235,193$.

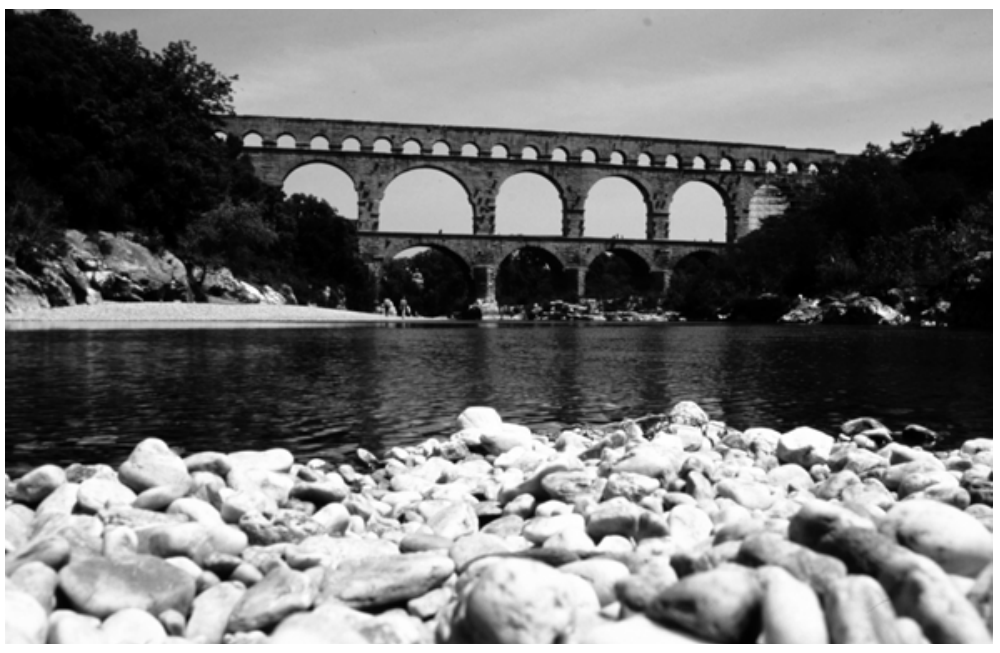

